Meta

Journal des traducteurs

Translators' Journal

\title{
MARTÍ FERRIOL, JOSÉ LUIS (2010) : Cine independiente y traducción. Valencia : Tirant Lo Blanch, 243 p.
}

\section{Valérie Florentin et Mayra Parra}

Volume 59, numéro 1, avril 2014

URI : https://id.erudit.org/iderudit/1026482ar

DOI : https://doi.org/10.7202/1026482ar

Aller au sommaire du numéro

\section{Éditeur(s)}

Les Presses de l'Université de Montréal

\section{ISSN}

0026-0452 (imprimé)

1492-1421 (numérique)

Découvrir la revue

\section{Citer ce compte rendu}

Florentin, V. \& Parra, M. (2014). Compte rendu de [MARTí FerRIol, JosÉ LuIS (2010) : Cine independiente y traducción. Valencia : Tirant Lo Blanch, 243 p.]

Meta, 59(1), 224-226. https://doi.org/10.7202/1026482ar d'utilisation que vous pouvez consulter en ligne.

https://apropos.erudit.org/fr/usagers/politique-dutilisation/ 
le plan de la concision, je dirais qu'on bat ici les ouvrages classiques puisqu'on donne à la fois les termes justes et les faux-amis en un peu plus d'une centaine de caractères.

Grant Hamilton adopte aussi à l'occasion un simple rôle d'observateur de la langue. Ainsi, lorsqu'il traite de la différence entre several et many, il se contente de dire qu'au Québec, plusieurs signifie souvent many alors qu'en France, il signifie several. Un avis légèrement plus critique aurait été apprécié. Par contre, on peut saluer son désir de décrire l'évolution de la langue, au tweet 587 par exemple, où il note la perte de popularité de shall en contexte juridique. Enfin, Grant Hamilton peut parfois se justifier en ayant recours au nombre d'occurrences dans Internet: dans le tweet 368, Offrir un cadeau, notamment, il justifie par des chiffres sa recommandation d'utiliser to give gifts, au lieu de to offer gifts. Ici encore, on peut cependant déplorer qu'il n'ait pas relevé le fait qu'en français non plus, on n'offre pas de cadeaux.

Fidèle à l'objectif qu'il se donne dans son avant-propos, ouvrir des portes, «donner des ailes et non les couper», François Lavallée dévoile davantage son esprit de pédagogue. Les solutions sont donc parfois moins pratiques et directement applicables que celles de son collègue anglophone. Ainsi, l'entrée 1345, «Tirer les marrons du feu n'est pas du tout la même chose que tirer son épingle du jeu ! », oblige le lecteur à une étape supplémentaire. François Lavallée a aussi à l'évidence une tendance plus marquée à user d'un esprit ludique, n'hésitant pas, par exemple, à se désigner comme "votre humble serviteur».

Moins nombreuses que chez Grant Hamilton, ses catégories sont aussi moins précises. Comment distinguer «Élaguons» de "Simplifions» et "Ouvrons nos horizons» de «Soyons ouverts», par exemple. Par ailleurs, on trouve des équivalences précises dans la section "Le mot juste», mais ailleurs également, bien sûr.

François Lavallée prend davantage position lorsqu'il s'agit de souligner des emplois abusifs dans la langue de départ. Ainsi, à l'entrée 1 253, après avoir signalé le risque de confusion entre e.g. et i.e., il ne se gêne pas pour ajouter que «les auteurs anglophones les confondent parfois eux-mêmes».

Comment utiliser Tweets et gazouillis? Je crois que Grant Hamilton a trouvé le mot juste dans sa préface: «I hope you have as much enjoyment perusing them as we had putting them together. " (c'est moi qui souligne).

Enfin, la question qui tue: le contenu d'ouvrages de ce genre étant accessible sur des fils Twitter, leur publication en format papier demeuret-elle pertinente? Il peut être utile de préciser ici que certains chercheurs universitaires remettent désormais en question la publication simultanée de versions papier et numérique de leurs ouvrages. C'est ce que me confiait récemment un chercheur dans un tout autre domaine que la traduction qui a de nombreuses publications à son actif. Or, on se trouve ici devant un cas de publication en version papier d'un contenu déjà diffusé en version numérique. Disons toutefois qu'en dépit du nombre de bénéficiaires des conseils et réflexions de ces deux réviseurs chevronnés sur leur fil respectif, il reste évidemment encore un bien plus grand nombre de traducteurs qui n'ont pas (encore) pris l'habitude de les consulter. Comme j'en fais partie, je me dis qu'après tout, nous le valons bien!

\section{Michel Buttiens, trad. A.} Montréal, Québec

\section{NOTES}

1. Deglise, Fabien (2 février 2012): Une époque obsédée par la concision et les formats courts. Le Devoir. p. F2.

2. Lavallée, François (2005): Le traducteur averti. Brossard: Linguatech.

3. Hamilton, Grant (2011): Les trucs d'anglais qu'on a oublié de vous enseigner. Québec: l'Instant même.

Martí Ferriol, José Luis (2010): Cine independiente y traducción. Valencia: Tirant Lo Blanch, $243 \mathrm{p}$.

Cette monographie se propose de comparer doublage et sous-titrage des films américains indépendants et présente, de fait, une étude de cas basée sur un corpus de cinq films, soit $\grave{A}$ l'ombre de la haine (Monsters' Ball, Marc Foster, 2001), Sans issue (In the Bedroom, Todd Field, 2001), Les heures (The Hours, Stephen Daldry, 2002), Eléphant (Elephant, Gus Van Sant, 2003) et Traduction infidèle (Lost in Translation, Sofia Coppola, 2003).

Dans le prologue de Frederic Chaume, celuici avance, dès le titre, que les modes de traduction ne seraient pas simplement deux modes de visionnement d'un film, mais plutôt le visionnement de deux films très différents. Cette question, sans être nouvelle (voir notamment Pettit 2004 ou Ramière 2004), demeure essentielle et pique l'intérêt du lecteur.

Dans l'introduction, Martí Ferriol décrit son amour du cinéma indépendant, l'ayant poussé à étudier plus précisément ce genre filmique. Il souligne entre autres que l'un des avantages du genre est que les films indépendants bénéficient souvent de deux traductions avant même leur sortie en DVD. En effet, ils sont dans un premier temps sous-titrés et destinés plutôt à une clientèle «d'intellectuels». Or, cette élite, plus éduquée, premièrement, ne rechigne pas à lire les sous-titres ${ }^{1}$ 
et, deuxièmement - l'anglais ayant quasiment un statut de lingua franca - pourrait fort bien comprendre la version originale sans avoir besoin des sous-titres. Par la suite, certains de ces films indépendants retiendront l'attention d'une plus grande frange du public, notamment s'ils gagnent des prix dans des festivals prestigieux (ce qui est le cas de tous les films du corpus ${ }^{2}$ ), et bénéficieront d'une seconde traduction, cette fois-ci par doublage. En somme, en étudiant les films indépendants, le mode de traduction n'est pas le seul élément qui change: le public visé devrait être pris en compte, ce qui n'est pas le cas ici. L'hypothèse de base, qui est d'affirmer que l'on traduit différemment en sous-titrage qu'en doublage (vérité de La Palisse s'il en est, ne serait-ce que par les contraintes techniques) est donc mise à mal, vu le corpus retenu. Il nous semble que les blockbusters américains, par exemple, auraient été un meilleur choix pour étudier l'impact du mode de traduction, à l'exclusion de tout autre facteur.

Dans le premier chapitre, l'auteur s'attache plus spécifiquement à la notion de genre filmique et souligne que tous les films retenus sont des drames. Il fait valoir que le film indépendant est un genre en soi, vu qu'il est décrit par une certaine tonalité et qualité visuelle, par la volonté de montrer le monde tel qu'il est ou tel que le réalisateur l'envisage plutôt qu'une fiction. Il s'agit généralement de films à petit budget (et, à cet égard, soulignons que le film Les Heures bénéficie d'un budget équivalant au double des quatre autres films retenus). Pourtant, plus qu'un genre, les films indépendants représentent plutôt un sous-genre (terme que l'auteur utilise, par ailleurs), puisque les films retenus sont d'abord et avant tout des drames. Parmi les drames, ils appartiennent à un sous-genre particulier, ou plutôt à une approche cinématographique particulière: les drames indépendants. L'uniformité du corpus ne permet pas de savoir si le mode de traduction diffère au sein du genre dramatique (un drame indépendant est-il traduit différemment d'un drame à plus gros budget?) de même qu'il ne permet pas de déterminer si le sous-genre des films indépendants bénéficie d'un mode de traduction distinct (la traduction d'une comédie indépendante sera-t-elle faite selon les mêmes règles que la traduction d'un drame indépendant?). Poser cette dernière question, c'est déjà y répondre. Enfin, on regrette que l'auteur ne spécifie pas en quoi, selon lui du moins, la traduction des films indépendants serait différente.

Dans le deuxième chapitre, l'auteur s'intéresse aux méthodes, ou formes, de traduction. Il reconnaît d'entrée de jeu que, malgré les contraintes fort différentes inhérentes à chaque mode de traduction, les répliques peuvent être semblables. Il bâtit ensuite son propre modèle d'analyse, en se basant, curieusement, sur des auteurs de langue espagnole uniquement, et regroupe en restrictions, normes et techniques ce que tout débutant connaît sous le nom de contraintes linguistiques, matérielles et temporelles. Il y ajoute cependant deux catégories: les restrictions nulles (donc l'absence, rarissime, de toute restriction) et les restrictions professionnelles (c'est-à-dire les exigences propres au client), rarement mentionnées mais déjà étudiées (notamment dans Gambier 2002, qui montre plusieurs exemples de la censure imposée aux traducteurs audiovisuels). Il est cependant regrettable que cette partie ne soit pas plus développée, plusieurs traducteurs contactés ayant refusé de répondre aux questions. En effet, il est rare que le travail des traducteurs soit analysé de l'intérieur et leur opinion aurait sûrement mieux permis de comprendre la différence quant au travail effectué.

Dans le troisième chapitre, l'auteur décrit sa méthode de travail. La présence de cette partie étonne et laisse croire que cette monographie était à l'origine une thèse de doctorat. Cela expliquerait aussi le style d'écriture, scolaire et répétitif, où l'auteur annonce ce qu'il compte faire, décrit ses résultats puis les résume en conclusion.

Le quatrième chapitre représente à lui seul trente pour cent de l'ouvrage et l'auteur s'y livre, dans le détail, à l'analyse proprement dite de son corpus. Les exemples y sont nombreux, mais les conclusions sont trop souvent d'une banalité effarante, notamment quand l'auteur constate que, dans le cas de dialogues rapides, les sous-titres sont condensés (p. 135), que sous-titrage comme doublage ont tendance à naturaliser ${ }^{3}$ et à expliciter ${ }^{4}$ les dialogues (p. 141). L'auteur souligne aussi que les gros mots sont souvent neutralisés à l'écrit, puisqu'ils seraient plus vulgaires à l'écrit qu'à l'oral (p. 161). Cette constatation est basée sur un seul exemple, mais concorde avec l'article de Gambier précité.

Le cinquième chapitre décrit d'une manière assez désordonnée les résultats obtenus. L'auteur revient premièrement sur les entrevues qu'il a obtenues avec certains des traducteurs concernés avant de reprendre les résultats mentionnés au chapitre précédent. Les dernières phrases du livre indiquent que l'auteur pense, après son étude, que voir le film sous-titré et voir le film doublé, ce n'est pas voir le même film. Si nous avons déjà fait part de nos réserves vu le corpus, il faut néanmoins ajouter à cela un fait de taille: l'auteur ne peut pas avoir une opinion sur le sujet, sa vision du film étant nécessairement faussée par l'étude réalisée certes, mais avant tout, par le fait que, au moment où il visionne le film pour la seconde fois dans un nouveau mode de traduction, sa perception est faussée par sa connaissance préalable du film. Il est en effet impossible de regarder le film à deux 
reprises et d'essayer de distinguer ce que nous avons perçu la première fois de ce que nous a apporté le deuxième visionnement.

Bref, si l'étude peut inspirer un chercheur débutant en quête d'un modèle d'étude de cas (celle-ci étant fort bien faite sur le plan méthodologique), sa lecture est fastidieuse pour tout chercheur quelque peu expérimenté, qui n’y trouvera pas son compte, la traduction des films indépendants n'étant visiblement pas un exercice différent au sein de la traduction audiovisuelle.

VALÉRIE FLORENTIN Université Laval, Québec, Canada

Mayra PArra

Université de Montréal, Montréal, Canada

\section{NOTES}

1. Plusieurs études réalisées pour la télévision, dont celle de Media Consulting Group en 2007, Les besoins et les pratiques de l'industrie audiovisuelle européenne en matière de doublage et de sous-titrage, démontrent qu'une émission sous-titrée peut perdre jusqu'à trente pour cent de son audience, les spectateurs n'étant pas habitués au sous-titrage.

2. Entre autres, Halle Berry a eu l'Oscar de la meilleure actrice pour À l'ombre de la haine; Sissy Spacek a eu le Golden Globe de la meilleure actrice pour Sans issue; Nicole Kidman a eu l'Oscar de la meilleure actrice pour Les heures; Éléphant a eu la Palme d'or à Cannes; Traduction infidèle a eu l'Oscar du meilleur scénario original.

3. C'est un fait reconnu et souvent étudié que, le canal visuel demeurant intact (Delabastita 1989), ce sont les autres canaux du texte polysémiotique qu'est le film qui font l'objet d'une adaptation. Les références, notamment culturelles, ne pouvant faire l'objet d'une explicitation vu les contraintes techniques, sont alors adaptées au public (donc, naturalisées). De nombreuses études de cas sont disponibles, dont plusieurs de Teresa Tomaszkiewicz. À l'inverse, une étude plus récente semble vouloir démontrer que les références culturelles sont généralement sauvegardées (Valentini 2011).

4. L'explicitation en traduction est, une fois de plus, une tendance reconnue et largement documentée depuis Blum-Kulka 1986. Par ailleurs, l'explication pragmatique décrite par Pym (1993) n'est rien d'autre qu'une naturalisation.

\section{RÉFÉRENCES}

Blum-KulKa, Shoshana (1986): Shifts of Cohesion and Coherence in Translation. In: Juliane House et Shoshana BLuM-KulKA, dir. Interlingual and Intercultural Communication: Discourse and Cognition in Translation and Second Language Acquisition Studies, 17-35.
Delabastita, Dirk (1989): Translation and Masscommunication: Film and TV Translation as Evidence of Cultural Dynamics. Babel. 35(4): 193-218.

GAmbier, Yves (2002): Les censures dans la traduction audiovisuelle. TTR. 15(2):203-221.

Pettit, Zoe (2004) : The Audio-visual Text: Subtitling and Dubbing Different Genres. Meta. 49(1):25-38.

Pym, Anthony (1993): Epistemological Problems in Translation and its Teaching: A Seminar for Thinking Students. Teruel, Spain: Edicions Caminade.

RAMIÈRE, Nathalie (2004): Comment le sous-titrage et le doublage peuvent modifier la perception d'un film. Meta. 49(1):102-114.

VAlentini, Cristina (2011): La traduction des références culturelles. In: Jean-Marc LAVAur et Adriana Şerban, dir. Traduction et médias audiovisuels. Lille: Septentrion Presses universitaires, 93-110.

BARAmbones Zubiria, Josu (2012): Lenguas minoritarias y traducción: La traducción audiovisual en euskera. Colección TRAMA. Castelló de la Plana: Publicacions de la Universitat Jaume I, 172 p.

Josu Barambones nos introduce en este libro en un campo todavía poco explorado: la traducción audiovisual (TAV) en euskera, lengua sobre la que las investigaciones y publicaciones en este ámbito brillan, aún hoy, por su ausencia. Esta obra resulta tremendamente necesaria, innovadora y útil para los estudios de TAV. Necesaria e innovadora por el ámbito escogido para el estudio: la traducción audiovisual en euskera, una lengua con menos de un millón y medio de hablantes, seis dialectos distintos y aún en proceso de normalización. Se aprecia una notable carencia de estudios descriptivos y empíricos que den cuenta de la situación actual de esta lengua minoritaria y del aporte que la práctica de la TAV ha supuesto en ella, en su cultura y en sus hablantes. Y útil por la metodología utilizada, fácilmente trasladable a otros estudios descriptivos en TAV basados en corpus.

El volumen está estructurado en siete capítulos. Los dos primeros suponen una introducción a la historia y la relación actual que la lengua vasca mantiene con la televisión y la TAV. Los restantes cinco capítulos acercan al lector a la tesis doctoral de Barambones, que, por un lado, pretende arrojar luz sobre las normas y técnicas empleadas en la traducción para doblaje de inglés a euskera y, por otro lado, constituye un análisis contrastivo de los modelos lingüísticos de textos audiovisuales de producción propia en euskera y de producción ajena doblados al euskera. 